\title{
Repercusión de bacterias lácticas y levaduras sobre el comportamiento productivo y salud de lechones
}

\section{Repercussion of lactic bacteria and yeasts on the productive performance and health of piglets}

José E. Miranda-Yuquilema ${ }^{1,2,4}$, Alfredo Marín-Cárdenas², Leonel Lazo-Pérez ${ }^{2}$, Davinia Sánchez-Macías ${ }^{3}$

\section{Resumen}

El objetivo del estudio fue evaluar el efecto de dos preparados microbianos en el comportamiento productivo, salud y perfil hematoquímico de lechones. Se emplearon 120 lechones (Duroc x Yorkshire/Landrace), crías de 12 cerdas reproductoras (Landrace/Yorkshire), que fueron distribuidos en tres grupos de 40 animales cada uno bajo un diseño completamente aleatorizado: control (T1); Biopreparado (T2) y Biopreparado (T3). El T2 contenía Lactobacillus acidophilus y Streptoccus thermophilus y T3 Saccharomyces cerevisiae y Kluyveromyces fragilis (L-4 UCLV). Se evaluó el peso al nacer, y el peso y ganancia de peso (GPV) en los días 14 y 33 (destete) de edad, así como la incidencia de diarreas, perfiles hemáticos y bioquímica sanguínea. El peso al nacer y la GPV de los lechones de T2 y T3 fue mayor que en los lechones del grupo control $(\mathrm{p}<0.05)$, sin variación entre T2 y T3. La ocurrencia de diarreas se redujo en $52 \%$ en los animales tratados con los probióticos. El perfil hematoquímico difirió entre tratamientos $(\mathrm{p}<0.05)$. Se concluye que el uso de bacterias lácticas y levaduras produjo un efecto positivo en el comportamiento productivo, salud y perfil hematoquímico de los lechones.

Palabras clave: probiótico; prebiótico; salud; residuo agroindustrial; producción porcina

\footnotetext{
${ }^{1}$ Instituto Nacional de Biodiversidad, Quito, Ecuador

${ }^{2}$ Facultad de Ciencias Agropecuarias, Universidad Central «Marta Abreu» de Las Villas, Cuba

${ }^{3}$ Facultad de Ingeniería, Universidad Nacional de Chimborazo, Ecuador

${ }^{4}$ E-mail: efra_miranda@outlook.com

Artículo derivado de un trabajo de tesis doctoral
}

Recibido: 29 de enero de 2018

Aceptado para publicación: 30 de junio de 2018 
The objective of this study was to evaluate the effect of two microbial preparations on the productive performance, health and blood profile of piglets. In total, 120 piglets (Duroc x Yorkshire/Landrace) offspring of 12 breeding sows (Landrace/Yorkshire) were distributed in three groups of 40 animals each under a completely randomized design: control (T1); Bioprepared (T2) and Bioprepared (T3). T2 contained Lactobacillus acidophilus and Streptoccus thermophilus and T3 Saccharomyces cerevisiae and Kluyveromyces fragilis (L-4 UCLV). Birth weight and body weight and body weight gain (BWG) on days 14 and 33 (weaning) of age, incidence of diarrhoea and blood and biochemical profiles were evaluated. The birth weight and the BWG of piglets of T2 and T3 was greater than in the piglets of the control group $(p<0.05)$, without difference between $\mathrm{T} 2$ and $\mathrm{T} 3$. The occurrence of diarrhoea was reduced by $52 \%$ in the animals treated with the probiotics. The hematochemical profile differed between treatments $(p<0.05)$. It is concluded that the use of lactic-acid bacteria and yeast produced a positive effect on the productive performance, health, and blood profile in piglets.

Key words: probiotic; prebiotic; health; agroindustrial waste; pig production

\section{INTRODUCCIÓN}

La población mundial se está incrementado rápidamente, y con ello, la demanda de alimentos de origen animal (FAO, 2016), lo que implica que los sistemas pecuarios deben incrementar la producción para suplir esta demanda de forma sustentable y amigable con el medio ambiente (Miranda et al., 2018). En las décadas pasadas, el método de mayor uso para prevenir enfermedades entéricas y aumentar la producción fue mediante el uso de antibióticos promotores de crecimiento como suplementos dietéticos; sin embargo, diversos estudios demuestran efectos negativos de estos en la eubiosis del sistema gastrointestinal, dando lugar a la aparición de resistencia bacteriana a fármacos y su presencia residual en los productos finales (carnes, leche, huevos, etc.) de origen animal, afectando así la salud humana al consumir estos productos (Datt et al., 2011; Liu et al., 2013; FAO, 2016). Esta situación ha hecho necesario introducir los probióticos como una alternativa ante esta problemática (Sun et al., 2015).
Una alternativa para aumentar el rendimiento productivo sin alterar el estado fisiológico del cerdo podría ser la utilización de microorganismos probióticos en la dieta basal (Rondón et al., 2013). Esto debido a su capacidad en el mejoramiento de la microbiota natural en el tracto digestivo (Giang et al., 2012), de romper los polímeros presentes en los alimentos a moléculas más pequeñas (Datt et al., 2013) y de incrementar la degradación y digestibilidad de los nutrientes (Rondon et al., 2013). Como consecuencia a lo anterior, se puede incrementar el comportamiento productivo y mejorar la salud del animal (Datt $e t$ al., 2011).

La disponibilidad y costo de los aditivos probióticos en países en vía de desarrollo están limitados, lo que minimizan las utilidades para el pequeño y mediano productor (Ayala et al., 2012; Miranda et al., 2018). Sin embargo, los biopreparados desarrollados con subproductos de la agroindustria pudiera resultar adecuado para su empleo como probióticos en la producción porcina. En este sentido, entre las especies microbianas más utilizadas para la obtención de probióticos se 
encuentran Bifedobaceriun, Lactobacillus, Streptococcus, Sacharomyces y Kluyeveromyces (Ayala et al., 2012; Ciro et al., 2015; Miranda et al., 2018).

Los cultivos microbianos con capacidad probiótica han sido evaluados y utilizados en diferentes especies de animales con resultados alentadoras. Un ejemplo de esto es la reducción de trastornos diarreicos en los animales jóvenes al mejorar el sistema inmune (Tabasum et al., 2014). Así mismo, se ha utilizado para tratar lechones afectados por $E$. coli y Salmonella spp con resultados positivos (Pajarillo et al., 2014, Zapata et al., 2015) y se ha observado una mejora en la ganancia de peso (Giraldo et al., 2015). También se tienen reportes sobre la reducción del contenido de triglicéridos en sangre de cerdos (Londoño y Parra, 2015). Teniendo en cuenta estos antecedentes, el presente estudio tuvo como objetivo evaluar el efecto de dos biopreparados sobre el comportamiento productivo y la salud de lechones al suministrarlos a ellos y a sus madres.

\section{Materiales y Métodos}

\section{Lugar de Estudio}

El trabajo se realizó en la unidad de producción porcina Gahuijón Alto en Cantón Colta, Ecuador. La zona se encuentra a 3510 msnm, presenta una precipitación anual entre 500 y $1000 \mathrm{~mm}$, temperatura mínima de $3{ }^{\circ} \mathrm{C}$, máxima de $14{ }^{\circ} \mathrm{C}$, media de $10{ }^{\circ} \mathrm{C}$, humedad relativa anual de $80 \%$ y evapotranspiración anual de $69.03 \mathrm{~mm}$.

\section{Tratamientos Experimentales}

Se utilizó un diseño completamente aleatorizado con cuatro repeticiones por tratamiento, donde cada unidad experimental estuvo compuesta por 12 animales. Los tratamientos evaluados fueron control (T1), biopreparado (T2) y biopreparado (T3).

\section{Animales y Dieta Basal}

Se emplearon cuatro cerdas reproductoras Landrace/Yorshire de segundo parto por tratamiento que fueron cruzadas con un cerdo Duroc. Luego del parto, 120 lechones fueron distribuidos al azar (sin alterar los grupos de procedencia) en los tres grupos experimentales (20 hembras y 20 machos por tratamiento).

Las cerdas madres estuvieron alojadas en corrales colectivos de $6 \times 6.5 \mathrm{~m}$ y piso de cemento, con una densidad de $1.8 \mathrm{~m}^{2}$ por animal desde la monta hasta los 110 días de gestación, donde pasaron a corrales individualess (maternidades). El alimento utilizado para todos los animales fue Bioalimentar ${ }^{\circledR}$ (Ambato, Ecuador), que cumple con los requerimientos nutricionales para cerdos recomendados por el NRC (2012). El alimento de los lechones estuvo basado en la leche materna y a partir del día siete posterior al nacimiento hasta el destete se suplementó ad libitum con alimento balaceado para lechones categoría predestete. El agua fue ofrecida ad libitum en bebederos tipo tetinas.

La maternidad se mantuvo a $28^{\circ} \mathrm{C} \mathrm{du}$ rante las dos primeras semanas posparto, y luego se redujo en $1.5^{\circ} \mathrm{C}$ por semana hasta el destete (33 días). El fotoperiodo fue controlado con 12 horas de luz y 12 horas de oscuridad. Las camadas de cada tratamiento se ubicaron distantes unas de otras, con un cuartón intermedio a ambos lados del pasillo, para evitar la autoinoculación. Los lechones recibieron las atenciones veterinarias pertinentes según el Manual de Manejo de Hembras y Primerizas (Coates et al., 2013).

\section{Preparados Microbianos}

Las cepas utilizadas fueron Kluyveromyces fragilis (L-4 UCLV) proveniente del Banco de Microorganismos de la Universidad Central «Marta Abreu» de Las Villas y tres cepas ATCC (American Type Cultures Collection, EEUU): Lactobacillus acido- 
philus, Streptococcus thermophilus y Saccharomyces cerevisiae. Las cepas fueron activadas en leche descremada a $37{ }^{\circ} \mathrm{C}$ por $24 \mathrm{~h}$. Para la obtención de los preparados se utilizó como sustrato la mezcla de melaza de caña de azúcar y vinaza de naranja y se fermentó a $37^{\circ} \mathrm{C}$ por $24 \mathrm{~h}$, según la metodología descrita por Miranda et al. (2018). En el biopreparado (T2) se emplearon las bacterias $L$. acidophilus más $S$. thermophilus y en el biopreparado (T3) se utilizaron $S$. cerevisiae más $K$. fragilis (L-4 UCLV). La composición química y la concentración microbiana de cada preparado se presentan en la Cuadro 1.

Los biopreparados se suministraron a las cerdas madres de los grupos T2 y T3 cada tres días a las 07:00. Para esto, se emplearon $20 \mathrm{ml}$ del aditivo microbiano asignado mezclado en $0.3 \mathrm{~kg}$ de dieta balanceado más $500 \mathrm{ml}$ de agua a partir de 30 días antes del parto hasta el destete. Las crías continuaron recibiendo el mismo aditivo que sus madres. El grupo control recibió suero fisiológico en igual cantidad que los grupos tratados.

\section{Variables de Respuesta}

Indicadores productivos. Los lechones fueron pesados al nacimiento, y a los 14 y 33 días de edad, calculándose la ganancia de peso (GP) y la ganancia media diaria (GMD) por edad y sexo. Para el pesaje se utilizó una báscula manual romana (URKO M95460, Suiza) de $20 \pm 0.05 \mathrm{~kg}$.

Diarreas y mortalidad. Se realizó un riguroso control clínico para detectar cambios de conducta, trastornos diarreicos y muertes. Se determinó la ocurrencia de diarrea y el porcentaje de mortalidad.

Perfil hematoquímico en sangre. Se seleccionaron al azar 12 lechones de cada tratamiento, se inmovilizaron y se extrajo $6 \mathrm{ml}$ de sangre de la vena yugular a los 14 y 33 días de edad. Las muestras se tomaron en tubos Vacutainer ${ }^{\circledR}$, con y sin etilendiamino- tetraacético (EDTA) con aguja tipo California y se trasladaron al laboratorio dentro de las tres primeras horas para su posterior procesamiento. Así mismo, se determinó hemoglobina $(\mathrm{Hb})$, hematocrito, número de eritrocitos, volumen corpuscular medio (VCM), hemoglobina corpuscular media (HCM) y concentración corpuscular media (CHCM), mediante la metodología descrita por Corredor (2012). El perfil químico evaluado fue: proteínas totales, triglicéridos, lipoproteína de alta densidad (HDL) y lipoproteína de baja densidad (LDL), según la metodología referida por Mejía et al. (2012).

\section{Análisis Estadístico}

Los datos experimentales se procesaron con el paquete estadístico Statgraphics Plus 15.1 para Windows. Se realizó análisis de varianza según diseño completamente aleatorizado y, en los casos necesarios, se aplicó la dócima de comparación de Duncan (1955) para discriminar diferencias entre medias a $\mathrm{p}<0.05$.

\section{Resultados}

En el Cuadro 2 se resumen los valores del comportamiento productivo de los lechones. El peso vivo (PV) en todas las edades y en ambos sexos fue menor en T1 en comparación con T2 y T3 $(\mathrm{p}<0.05)$, sin variación entre estos últimos. La GMD fue mayor en los grupos tratados con biopreparados que contenían bacterias lácticas (T2) y levaduras (T3) frente al tratamiento control $(\mathrm{p}<0.05)$. El PV de los animales de T2 y T3 a los 14 días fue superior en $1.8 \mathrm{~kg}$ (machos) y $1.0 \mathrm{~kg}$ (hembras) sobre el grupo control, y de 2.35 $\mathrm{kg}$ en ambos sexos al destete.

En la Figura 1 se presenta la ocurrencia semanal de diarreas desde el nacimiento hasta el destete. Se observó una menor ocurrencia en los animales tratados con los biopreparados (T2 y T3) con respecto a los del grupo control 
Cuadro 1. Composición bromatológica y microbiológica de los biopreparados

\begin{tabular}{lcc}
\hline \multirow{2}{*}{ Indicadores } & Biopreparado & Biopreparado \\
& T2 & T3 \\
\hline Materia seca, \% & 17.5 & 18.5 \\
Proteína cruda, \% & 17.2 & 17.7 \\
Proteína verdadera, \% & 12.3 & 12.5 \\
Extracto etéreo, \% & 2.83 & 2.43 \\
Cenizas, \% & 2.12 & 2.26 \\
pH & 3.86 & 3.85 \\
Ácido láctico, mmol/1 & 0.72 & 0.74 \\
Concentración microbiana, UFC/ml & $9.4 \times 10^{8}$ & $9.5 \times 10^{9}$ \\
\hline
\end{tabular}

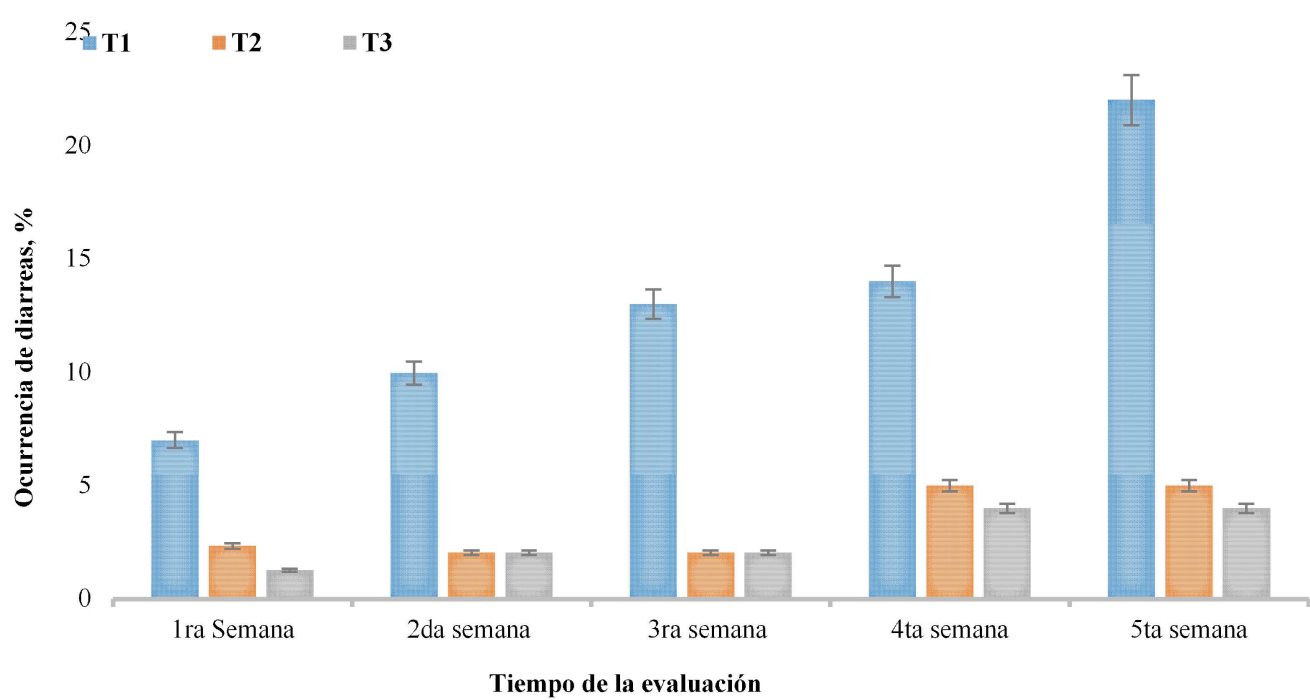

Figura 1. Ocurrencia de diarreas en los lechones entre el nacimiento y el destete (33 días). T1, Dieta basal sin aditivo. T2, L. acidophilus y $S$. thermophilus. T3, S. cerevisiae y $K$. fragilis (L-4 UCLV)

$(\mathrm{p}<0.05)$. En las semanas 4 y 5 hubo aumento de trastornos diarreicos y muertes en $\mathrm{T} 1$.

En el Cuadro 3 se observa el perfil hemático de los lechones a los 14 y 33 días de edad. Los valores de la hemoglobina de los lechones tratados (T2 y T3) fueron mayores con respecto al grupo control, en am- bas mediciones $(\mathrm{p}<0.05)$. El hematocrito no difirió significativamente entre tratamientos a los 14 días, pero fue menor a los 33 días en T1 frente a T2 y T3 $(\mathrm{p}<0.05)$; así mismo, los valores de VCM, HCM y CHCM no difirieron entre tratamientos a los 14 días, mientras que fueron mayores al destete en $\mathrm{T} 1$ frente a T2 y T3 $(\mathrm{p}<0.05)$. 
Cuadro 2. Comportamiento de peso vivo y ganancia media diaria de lechones hijos de madres suplementadas con dos tipos de biopreparados

\begin{tabular}{|c|c|c|c|c|c|c|c|}
\hline \multirow{2}{*}{ Edad } & \multirow{2}{*}{ Indicadores } & \multirow{2}{*}{ Sexo } & \multicolumn{3}{|c|}{ Tratamientos } & \multirow{2}{*}{$\pm \mathrm{EE}$} & \multirow{2}{*}{ valor- $p$} \\
\hline & & & $\mathrm{T} 1$ & $\mathrm{~T} 2$ & $\mathrm{~T} 3$ & & \\
\hline \multirow[t]{2}{*}{$1 \mathrm{~d}$} & \multirow[t]{2}{*}{$\mathrm{PV}, \mathrm{kg}$} & M & $1.10^{\mathrm{b}}$ & $1.35^{\mathrm{a}}$ & $1.40^{\mathrm{a}}$ & 0.06 & 0.0101 \\
\hline & & $\mathrm{H}$ & $1.05^{\mathrm{b}}$ & $1.15^{\mathrm{a}}$ & $1.25^{\mathrm{a}}$ & 0.01 & 0.0125 \\
\hline \multirow[t]{4}{*}{$14 \mathrm{~d}$} & \multirow[t]{2}{*}{$\mathrm{PV}, \mathrm{kg}$} & M & $3.70^{\mathrm{b}}$ & $4.85^{\mathrm{a}}$ & $4.91^{\mathrm{a}}$ & 0.02 & 0.0125 \\
\hline & & $\mathrm{H}$ & $3.43^{\mathrm{b}}$ & $4.26^{\mathrm{a}}$ & $4.55^{\mathrm{a}}$ & 0.02 & 0.0125 \\
\hline & \multirow[t]{2}{*}{ GMD, $\mathrm{g}$} & M & $264^{\mathrm{b}}$ & $346^{\mathrm{a}}$ & $350^{\mathrm{a}}$ & 0.01 & $<.0001$ \\
\hline & & $\mathrm{H}$ & $245^{\mathrm{b}}$ & $304^{\mathrm{a}}$ & $325^{\mathrm{a}}$ & 0.21 & 0.0012 \\
\hline \multirow[t]{4}{*}{$33 \mathrm{~d}$} & \multirow[t]{2}{*}{$\mathrm{PV}, \mathrm{kg}$} & M & $9.40^{\mathrm{b}}$ & $11.85^{\mathrm{a}}$ & $11.95^{\mathrm{a}}$ & 0.08 & 0.0122 \\
\hline & & $\mathrm{H}$ & $9.25^{\mathrm{b}}$ & $11.55^{\mathrm{a}}$ & $11.65^{\mathrm{a}}$ & 0.02 & $<.0001$ \\
\hline & \multirow[t]{2}{*}{ GMD, $\mathrm{g}$} & M & $522^{\mathrm{b}}$ & $658^{\mathrm{a}}$ & $663^{\mathrm{a}}$ & 0.01 & 0.0242 \\
\hline & & $\mathrm{H}$ & $513^{\mathrm{b}}$ & $641^{\mathrm{a}}$ & $647^{\mathrm{a}}$ & 0.01 & $<.0001$ \\
\hline
\end{tabular}

a,b,c Letras distintas en la misma fila difieren significativamente $(\mathrm{p}<0.05)$

PV: peso vivo, GMD: ganancia media diaria de peso, d: días, M: macho, H: hembra

T1: dieta basal sin aditivo, T2: L. acidophilus y S. thermophilus, T3: S. cerevisiae y K. fragilis (L-4 UCLV)

Cuadro 3. Perfil hemático a los 14 y 33 días de edad de lechones hijos de cerdas suplementadas con biopreparados (T2 y T3) desde 30 días preparto hasta el destete (33 días posparto)

\begin{tabular}{|c|c|c|c|c|c|c|}
\hline \multirow{2}{*}{ Parámetros } & \multirow{2}{*}{$\begin{array}{l}\text { Edad } \\
\text { (días) }\end{array}$} & \multicolumn{3}{|c|}{ Tratamientos } & \multirow{2}{*}{$\pm \mathrm{EE}$} & \multirow{2}{*}{ valor- $p$} \\
\hline & & $\mathrm{T} 1$ & $\mathrm{~T} 2$ & $\mathrm{~T} 3$ & & \\
\hline \multirow{2}{*}{ Hemoglobina, $g / 1$} & 14 & $108.5^{b}$ & $110.4^{\mathrm{a}}$ & $111.8^{\mathrm{a}}$ & 0.08 & 0.0130 \\
\hline & 33 & $110.7^{b}$ & $112.6^{\mathrm{a}}$ & $112.4^{\mathrm{a}}$ & 0.02 & 0.0070 \\
\hline \multirow{2}{*}{ Hematocrito, \% } & 14 & 0.28 & 0.29 & 0.28 & 0.01 & 1.1064 \\
\hline & 33 & $0.31^{\mathrm{b}}$ & $0.35^{\mathrm{a}}$ & $0.35^{\mathrm{a}}$ & 0.02 & 0.0121 \\
\hline \multirow{2}{*}{ VCM, fl } & 14 & 38 & 38 & 39 & 0.10 & 0.8129 \\
\hline & 33 & $35^{\mathrm{a}}$ & $32^{b}$ & $32^{b}$ & 0.06 & 0.0312 \\
\hline \multirow{2}{*}{ HCM, pg } & 14 & 38 & 38 & 39 & 0.07 & 0.6019 \\
\hline & 33 & $35^{\mathrm{a}}$ & $32^{\mathrm{b}}$ & $32^{b}$ & 0.32 & 0.0214 \\
\hline \multirow{2}{*}{ CHCM, g/l } & 14 & 387 & 380 & 399 & 0.22 & 0.5833 \\
\hline & 33 & $357^{\mathrm{a}}$ & $321^{\mathrm{b}}$ & $321^{\mathrm{b}}$ & 0.10 & 0.0121 \\
\hline
\end{tabular}

a,b,c Medias con superíndices diferentes indica diferencias significativas $(\mathrm{p}<0.05)$

T1: dieta basal sin aditivo, T2: L. acidophilus y S. thermophilus, T3: S. cerevisiae y K. fragilis (L-4 UCLV)

VCM: volumen corpuscular medio; HCM: hemoglobina corpuscular media; CHCM: concentración corpuscular media 
Cuadro 4. Comportamiento de la bioquímica sanguínea a los 14 y 33 días de edad de lechones hijos de cerdas suplementadas con biopreparados (T2 y T3) desde 30 días preparto hasta el destete (33 días posparto)

\begin{tabular}{lcccccc}
\hline \multirow{2}{*}{ Parámetros } & \multirow{2}{*}{ Edad, d } & \multicolumn{3}{c}{ Tratamientos } & \multirow{2}{*}{ \pm EE } & \multirow{2}{*}{ valor- $p$} \\
\cline { 3 - 5 } & & T1 & T2 & T3 & & \\
\hline Proteínas totales, & 14 & $48.05^{\mathrm{b}}$ & $53.42^{\mathrm{a}}$ & $53.68^{\mathrm{a}}$ & 0.12 & 0.0129 \\
$\mathrm{~g} / \mathrm{l}$ & 33 & $55.33^{\mathrm{b}}$ & $67.31^{\mathrm{a}}$ & $67.63^{\mathrm{a}}$ & 0.22 & 0.6312 \\
Triglicéridos, & 14 & $1.21^{\mathrm{a}}$ & $1.13^{\mathrm{b}}$ & $1.15^{\mathrm{b}}$ & 0.01 & 0.0064 \\
mmol/1 & 33 & $1.34^{\mathrm{a}}$ & $0.88^{\mathrm{b}}$ & $0.85^{\mathrm{b}}$ & 0.32 & 0.0121 \\
C-HDL, mmol/1 & 14 & $0.68^{\mathrm{b}}$ & $0.78^{\mathrm{a}}$ & $0.75^{\mathrm{a}}$ & 0.01 & 0.0197 \\
& 33 & $0.85^{\mathrm{b}}$ & $1.11^{\mathrm{a}}$ & $1.12^{\mathrm{a}}$ & 0.12 & 0.0124 \\
C-LDL, mmol/1 & 14 & $28.5^{\mathrm{a}}$ & $25.3^{\mathrm{b}}$ & $26.1^{\mathrm{b}}$ & 0.22 & 0.0312 \\
& 33 & $30.14^{\mathrm{a}}$ & $22.32^{\mathrm{b}}$ & $21.12^{\mathrm{b}}$ & 0.07 & 0.0019 \\
\hline
\end{tabular}

a,b,c Medias con superíndices diferentes indica diferencias significativas $(\mathrm{p}<0.05)$

T1: dieta basal sin aditivo, T2: L. acidophilus y S. thermophilus, T3: S. cerevisiae y K. fragilis (L-4 UCLV)

C-HDL: colesterol lipoproteína de baja densidad; C-LDL: lipoproteína de baja densidad

En el Cuadro 4 se observa el comportamiento de la bioquímica sanguínea de los lechones. La proteína total fue menor en T1 en comparación a los demás tratamientos en las dos mediciones $(\mathrm{p}<0.05)$. Los triglicéridos $\mathrm{y}$ LDL fue mayor para los animales del grupo control $(\mathrm{T} 1)(\mathrm{p}<0.05)$; sin embargo, se observó un efecto contrario para el indicador HDL en los lechones descendientes de las madres que no consumieron bacterias lácticas y levaduras (T1) frente a los demás tratamientos para ambas edades $(\mathrm{p}<0.05)$.

\section{Discusión}

El mejor peso de los lechones en las dos etapas del estudio fue posiblemente debido al efecto benéfico de los microorganismos empleados en la dieta de las madres. Estos resultados guardan relación con los reportados por Giang et al. (2012) y por Ayala et al. (2012), quienes con el suministro de probióticos en la dieta de las cerdas madres a partir del último tercio de la gestación lograron mejorar el comportamiento productivo de los lechones; por otro lado, los valores reportados por Zongyong et al. (2015) con dietas suplementadas con Saccharomyces cerevisiae fueron inferiores a los obtenidos en el presente estudio en lo que se refiere a la ganancia de peso. Esto demuestra que los cultivos mixtos de bacterias lácticas y levaduras desarrollados en subproductos de la agroindustria pueden contribuyen a una mayor asimilación de nutrientes y actuar como promotores de crecimiento en lechones.

En un estudio previo, Miranda et al. (2018) empleando un cultivo mixto de bacterias lácticas y levaduras en la dieta de cerdas reproductoras, lograron aumentar la cantidad de leche producida, teniendo por consecuencia una mejora en el peso de los lechones al destete. Sin embargo, los valores reportados por Liu et al. (2013) y Pajarillo et al. (2014) son inferiores a los obtenidos en el presente estudio. 
Por su parte, Ayala et al. (2012) con el suministro de Bacillus subtilis y sus endosporas reportaron mejorar la ganancia de peso en lechones y Mora et al. (2012) usando subproductos de la agroindustria reportan $232 \mathrm{~g} /$ día. Por el contrario, Veizaj-Delia et al. (2010) al emplear Lactobacillus plantarum, Lactobacillus fermentum y Enterococcus faecium obtuvieron valores superiores a $285 \mathrm{~g} /$ día, debido probablemente a que usaron un cultivo mixto en la alimentación. Estos resultados demuestran la importancia del uso de preparados microbianos a partir de bacterias lácticas y levaduras como probiótico de uso veterinario; así como al origen de los sustratos para la elaboración de los bioproductos y la composición nutricional de las raciones alimenticias. Los resultados obtenidos en el presente estudio coinciden con los trabajos de Tabasum et al. (2014) y Ciro et al. (2015), quienes argumentan que los cultivos mixtos de bacterias y levaduras mejoran los resultados productivos.

El suministro del cultivo mixto de bacterias (L. acidophilus y $S$. thermophilus) y levaduras (S. cerevisiae y K. fragilis L-4 UCLV) redujo la ocurrencia de diarreas debido a que estos actúan en el movimiento de iones y agua que siguen un gradiente osmótico a través de la pared intestinal (Farzan et al., 2013). De esta forma, el tracto digestivo está mejor preparado para enfrentar posibles agresiones por parte de los patógenos (Ciro et al., 2015). Por ejemplo, Datt et al. (2011), al adicionar probiótico en la dieta de los cerdos jóvenes, lograron reducir la incidencia de diarreas y la tasa de mortalidad; así mismo, Giang et al. (2012) mejoraron la salud de lechones al destete con dietas que contenían Bacillus subtilis y $S$. boulardii. Similares resultados fueron reportados por Liu et al. (2013) con L. rhamnosus GG. Estos resultados concuerdan con los planteamientos de Miranda et al. (2018) y Rondón et al. (2013), quienes mencionan que el uso de bacterias lácticas y lavaduras mejora la inmunidad, aumenta la producción de ácidos orgánicos de cadena corta, cambia de los valores de $\mathrm{pH}$ intestinal, mejora la digestibilidad, y limita o elimina los agentes patógenos promotores de las diarreas en animales jóvenes.

Los cambios en el perfil hemoquímico (Cuadros 3 y 4) no provocaron un efecto negativo en la salud de los animales, debido a que los valores de la referencia fisiológica estuvieron dentro de los rangos considerados normales para esta especie y categoría (Corredor, 2012). En este sentido, Mejía et al. (2012), Londoño y Parra (2015) y Liu et al. (2013) observaron una reducción de los niveles séricos al suplementar bacterias lácticas, lo que pudiera afirmar el efecto benéfico de los aditivos microbianos sobre la salud del animal (Ciro et al., 2015). Por otro lado, Pajarillo et al. (2014) y Sun et al. (2015) argumentan que el efecto probiótico de los microorganismos es debido a la capacidad de modificar la microbiota intestinal del huésped, lo que se traduce en el mejoramiento de la salud del huésped.

Otro de los efectos benéficos de los biopreparados reportados por Colina et al. (2011) y Londoño y Parra (2015) fue la acción sobre el plasma sanguíneo, al reducir los niveles del colesterol total, LDL y el aumento de HDL, pues mejora el funcionamiento de los órganos metabólicos (Colina et al., 2011; Mejía et al., 2012; Zapata et al., 2015).

\section{Conclusiones}

- La inclusión de bacterias lácticas y levaduras en la dieta básica de las cerdas madres a partir del último tercio de la gestación mejora el comportamiento productivo en la descendencia.

- Los trastornos diarreicos y muertes se reducen al suplementar preparados microbianos obtenidos a partir de bacterias lácticas y lavaduras.

- Los valores hematoquímicos de los cerdos a los 14 y 33 días de edad se encuentran en un mejor rango fisiológico favoreciendo la salud de los lechones. 


\section{Agradecimientos}

El autor principal agradece al Instituto de Fomento a Talento Humano (IFTH), Secretaría de Educación Superior Ciencia, Tecnología e Innovación (SENESCYT) por la beca de Cooperación Internacional otorgada para la formación de Doctor en Ciencias $(\mathrm{PhD})$. Así mismo, a la Facultad de Ciencias, Escuela Superior Politécnica de Chimborazo, Riobamba, Ecuador, por facilitar el uso de los equipos y los laboratorios de microbiología, clínica y bromatología para desarrollar el presente estudio.

\section{Literatura Citada}

1. Ayala L, Bocourt R, Milián G, Castro M, Herrera M, Guzmán J. 2012. Evaluación de un probiótico basado en Bacillus subtilis y sus endosporas en la obtención de pulmones sanos de cerdos. Rev Cubana Cienc Agríc 46: 391-394.

2. Ciro JA, López A, Parra J. 2015. La adición de cepas probióticas modula la secreción de mucinas intestinales en íleon de cerdos en crecimiento. Rev CES Med Vet Zootec 10: 150-159.

3. Coates J, Corns PJ, Juárez A, MacDonald R, McCulley N, Melody $B$, Minton A, et al. 2013. Manual PIC de manejo de hembras y primerizas. EEUU: PIC. 48 p.

4. Colina J, Méndez A, Araque H, Rueda E, León M, Rossini M. 2011. Lípidos sanguíneos en cerdos alimentados con pijiguao (Bactris gasipaes Kunth) y lisina sintética. Rev MVZ Córdoba 16: 2668-2677. doi: 10.21897/rmvz.267

5. Corredor R. 2012. Perfil hemático de cerdos alimentados con follaje de Morera Morus alba. Rev CITECSA 2: 27-35.

6. Datt C, Malik S, Datta M. 2011. Effect of probiotics supplementation on feed consumption, nutrient digestibility and growth performance in crossbred pigs under Tripura climate. Indian J Anim Nutr 28: 331-335.
7. Duncan DB. 1955. Multiple range and multiple F test. Biometrics 11: 1-42.

8. Bajagai YS, Klieve AV, Dart PJ, Bryden WL, FAO. 2016. Probiotics in animal nutrition: production, impact and regulation. FAO Anim Prod Health Paper No. 179. Rome: FAO. 89 p.

9. Farzan A, Kircanski J, Delay J, Soltes G, Songer JG, Friendship R, Prescott $J F$. 2013. An investigation into the association between $c p b 2$-encoding Clostridium perfringens type A and diarrhea in neonatal piglets. Can J Vet Res 77: 45-53.

10. Giang HH, Viet TQ, Ogle B, Lindberg $J$. 2012. Growth performance, digestibility, gut environment and health status in weaned piglets fed a diet supplemented with a complex of lactic acid bacteria alone or in combination with Bacillus subtilis and Saccharomyces boulardii. Livestock Sci 143: 132-141. doi: 10.1016/j.livsci.2011.09.003

11. Giraldo J, Narváez W, Díaz E. 2015. Probióticos en cerdos: resultados contradictorios. Rev Biosalud 14(1): 81-90. doi: 10.17151/biosa.2015.14.1.9

12. Liu F, Li G, Wen $K$, Wu S, Zhang Y, Bui T, Yang $X$, et al. 2013. Lactobacillus rhamnosus GG on rotavirus induced injury of ileal epithelium in gnotobiotic pigs. J Pediatr Gastr Nutr 57: 750-780. doi: 10.1097/MPG.0b0$13 \mathrm{e} 3182 \mathrm{a} 356 \mathrm{e} 1$

13. Londoño S, Parra J. 2015. Efecto de la adición de cepas probióticas sobre metabolitos sanguíneos en cerdos en crecimiento. Biotecnol Sector Agropec Agroind 13: 49-56.

14. Mejía K, Lemus C, Huerta R, Almague R, Ly J. 2012. Niveles séricos de triglicéridos y colesterol en cerdos cuino mexicano. Rev Comput Prod Porc (RCPP) 19: 37-41.

15. Miranda JE, Marin A, Oliva H, Baño, $D$, et al. 2018. Influence of a microbial additive on the productive behavior of pregnant sows, as well as, hematochemical and diarrheal incidence in their offspring. J.Trop Subtrop Agroecosist 21:39-45. 
16. Miranda JE, Marin A, Sánchez D, Hernandez Y. 2018. Elaboration of a bioprepared with probiotic effect from a mixed culture of lactic bacteria and yeasts. Bionatura 2: 245-247. doi: 10.21931/RB/2017.02.01.6

17. Mora LM, Lezcano P, Hidalgo K, Rodríguez B. 2012. Levadura torula (Candida utilis) en vinaza de destilería en dietas para cerdos en crecimiento. Rev Cubana Cienc Agr 46: 63-65.

18. NRC, National Research Council. 2012. Nutrient requirements of swine. $11^{\text {th }}$ rev. ed. Washington, DC: The National Academies. $450 \mathrm{p}$.

19. Pajarillo AB, Chae JP, Balolong M, Bum H, Kang DK. 2014. Assessment of fecal bacterial diversity among healthy piglets during the weaning transition. J Gen Appl Microbiol 60: 140-146. doi: 10.2323/jgam. 60.140

20. Rondón A, Ojito Y, Arteaga F, Laurencio M, Milián G, Pérez $Y$. 2013. Efecto probiótico de Lactobacillus salivarius $\mathrm{C} 65$ en indicadores productivos y de salud de cerdos lactantes. Rev Cubana Cienc Agríc 47: 401-407.

21. Sun Y, Park I, Guo J, Weaver A, Woo S. 2015. Impacts of low level aflatoxin in feed and the use of modified yeast cell wall extract on growth and health of nursery pigs. Anim Nutr 1: 177-183. doi: 10.1016/j.aninu.2015.08.012

22. Tabasum AS, Hoon J, Mun H, Yang C. 2014. Evaluation of Lactobacillus and Bacillus based probiotics as alternatives to antibiotics in enteric microbial challenged weaned piglets. Afr J Microbiol Res 8: 96-104. doi: 10.5897/ AJMR2013.6355

23. Veizaj-Delia E, Piu T, Lekajc P, Tafaj M. 2010. Using combined probiotic to improve growth performance of weaned piglets on extensive farm conditions. Livestock Sci 134: 249-251. doi: 10.1016/ j.livsci.2010.06.155

24. Zapata D, Rodríguez B, Ramírez M, López A, Parra J. 2015. Escherichia coli lipopolysaccharide affects intestinal mucin secretion in weaned pigs. Rev Colomb Cienc Pec 28: 209-217. doi: 10.17533/udea.rccp.v28n3a01.

25. Zongyong J, Shaoyong $W$, Zhilin $W$, Cui Z, Shenglan H, Chuntian Z, Zhuang C, et al. 2015. Effects of different forms of yeast Saccharomyces cerevisiae on growth performance, intestinal development, and systemic immunity in early-weaned piglets. J Anim Sci Biotechnol 6: 1-8. doi: 10.1186/ s40104-015-0046-8 